\title{
Intraoperative optical flow based tremor evaluation - a feasibility study
}

\author{
Hemm-Ode $\mathrm{S}^{1}$, Wettmann $\mathrm{P}^{1}$, Kistler $\mathrm{B}^{1}$, Behm $\mathrm{P}^{1}$, Schkommodau $\mathrm{E}^{1}$, Coste $\mathrm{J}^{2}$, Lemaire $\mathrm{J}^{2}$, Shah $\mathrm{A}^{1}$ \\ ${ }^{1}$ Institute for Medical and Analytical Technologies, School of Life Sciences, University of Applied Sciences and \\ Arts Northwestern Switzerland, Switzerland \\ ${ }^{2}$ Centre Hospitalier Universitaire de Clermont-Ferrand, Image-Guided Clinical Neurosciences and Connectomics \\ (EA 7292, IGCNC), Université d'Auvergne, France
}

Simone.hemm@fhnw.ch

\begin{abstract}
Deep brain stimulation as treatment for movement related disorders is a common neurosurgical procedure. Nevertheless the targeting procedure can still be optimized as the clinical outcome resulting from intraoperative test stimulation is in general subjectively evaluated. The aim of the present study was to analyse the feasibility of objective tremor evaluation based on optical flow analysis in videos and to compare the results with in parallel performed acceleration measurements. The results demonstrate the feasibility but as well limitations of the applied set-up. Solutions to increase the quality in the future are proposed.
\end{abstract}

Keywords: optical flow, deep brain stimulation, acceleration measurements, intraoperative tremor evaluation

\section{Introduction}

Deep brain stimulation (DBS) is a common neurosurgical procedure for relieving movement related disorders such as Parkinson's disease. DBS extends uncertainties associated with suboptimal target selection. In order to refine the target selected on the anatomical images, microelectrode recording and test stimulations are intraoperatively performed and subjectively evaluated.

The idea to objectively analyse tremor via video recording is not new. The motion between two image frames can be estimated by optical flow methods. Uhrikova has presented a tool for automatic detection and frequency measurement of tremor [1]. A comparison with accelerometer measurements showed closely related frequencies. Other authors used infrared LEDs attached to the body which are tracked by a simple CCD camera [2]. To our knowledge, no study has been published so far dealing with optical flow analysis for evaluating tremor in the operating room (OR). Consequently, the aim of the present study was to investigate the feasibility to evaluate tremor in the OR by optical flow analysis and to compare the data with in parallel recorded acceleration.

\section{Methods}

Patients: Two patients referred for bilateral DBSimplantation (1 Parkinson's Disease, 1 Essential Tremor) were included in the study after having given informed consent.

Surgical Procedure: Surgery was performed as described in [3]. After preoperative stereotactic MRI and CT acquisitions, the surgical planning was performed the day before surgery. The final implantation site was determined the day of surgery based on intraoperative microelectrode recording (MER) and test stimulation. Two trajectories were tested for each hemisphere. While the intensity of electric current used for stimulation was increased, changes in tremor amplitude were analysed by the neurosurgeon to determine the moment of and the amplitude at tremor improvement (surgeon threshold). During the evaluation, a 3-axis accelerometer (sampling rate $400 \mathrm{~Hz}$ ) synchronized with the stimulation amplitude, was fixed to the patient's wrist and the observed arm was filmed with a digital video camera (Canon LEGRIA FS200) on a tripod at a distance of approximately $2 \mathrm{~m}$. Resolution and recording frequency were $720 \times 576$ pixels and 25 frames/s respectively.

Data analysis: Synchronisation between video and acceleration data had to be performed manually. They were both grouped according to stimulation amplitudes. Interesting sequences were extracted from the video and software was developed to analyse the movement of the arm within a chosen region of interest (ROI) based on the Lucas-Kanade algorithm [4] implemented in Java (JavaCV using OpenCV 2.4.2): a vector field was calculated for the ROI based on the highest probability of movement of a $5 \times 5$ mask from one frame to the next (Fig. 1).

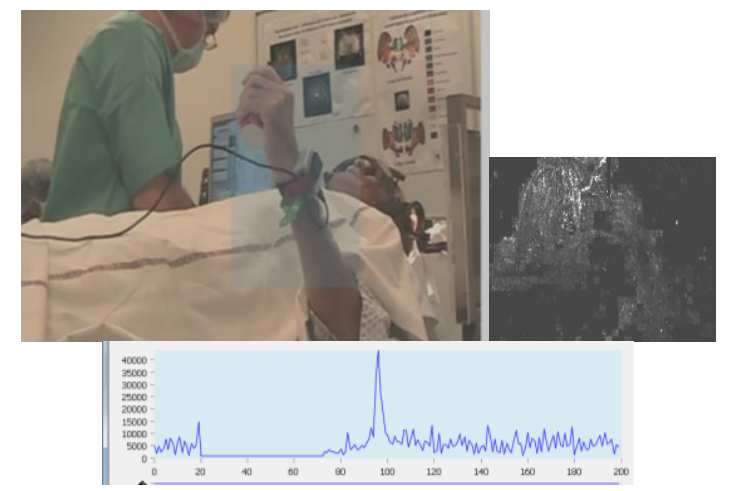

Figure 1: Top left: Scene with chosen analysis window (blue); top right: vector field between two frames; bottom: optical flow [arbitrary units] over time $[\mathrm{s}]$

The absolute values of the vectors were determined. To reduce camera specific noise, data were filtered with a median filter. Statistical features (standard deviation, energy, entropy) were extracted for each test stimulation position using Matlab (MathWorks Inc., Bern, Switzer- 
land) (Fig. 2). An optical flow specific threshold for significant clinical improvement was determined for each position: the percentage change of all three mathematical features compared to baseline recording without stimulation had to be of $75 \%$ or, if no such high improvement existed, of $50 \%$. The three features were statistically compared before and after this threshold (Wilcoxon signed rank test). Their correlation with acceleration data and the neurosurgeon's observations was performed visually and statistically (Spearman rank test).

\section{Results}

Synchronisation of acceleration and optical data could be performed for both patients (+/- 0.5 - 2 seconds). Statistical parameters were calculated for all positions and stimulation amplitudes. Optical, acceleration and surgeon's thresholds were compared. In all cases, tremor decrease corresponded to a clear decrease in statistical parameters. The visual comparison of optical flow and acceleration thresholds showed nearly similar trends for all trajectories (Fig. 2).

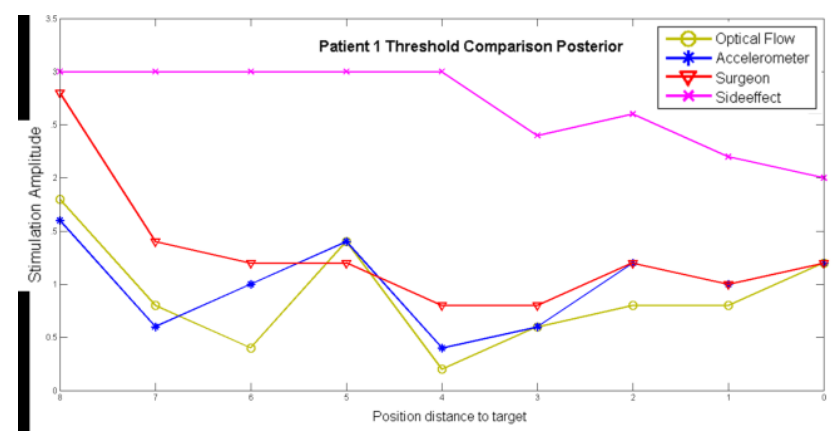

Figure 2: Threshold comparison - stimulation amplitude $[\mathrm{mA}]$ over the distance to the exploration endpoint [mm].

The statistical comparison between accelerometer and optical flow data for each position separately showed mostly high correlation in patient 1 and weaker correlation in patient 2. For patient 1 the optical flow thresholds were in general at or even below the surgeon's thresholds (Fig. 2). In patient 2 the optical flow threshold was in many cases higher than the surgeon's and the acceleration one.

\section{Discussion}

The aim of the present study was to analyse the feasibility to intraoperatively use video recordings for tremor analysis by comparing the results to accelerometer measurements. The accelerometer has the advantage that no line of sight problem exists but on the other hand it has to be connected by USB for use in the OR. Another weakness mentioned before [2] is that the sensors cannot satisfactorily track slow or constant speed motion where some of tremors may have significant spectral components.

The present study demonstrated the general feasibility of using video recording for tremor evaluation in the OR as a decrease in tremor is always visible as a decrease in the statistical features. Even if correlation between the two data types is not optimal, results are satisfying considering the fact that the analysis of video recording was not pre-planned and in consequence recording conditions have not been optimal. We propose different solutions for the identified limitations of the current set-up (Tab. 1).

Table 1: Solutions to identified limitations

\begin{tabular}{ll}
\hline Limitation & Possible solutions \\
\hline $\begin{array}{l}\text { Suboptimal camera posi- } \\
\text { tion }\end{array}$ & $\begin{array}{l}\text { Positioning closer to the } \\
\text { patient or optimally at the } \\
\text { ceiling }\end{array}$ \\
$\begin{array}{l}\text { Suboptimal synchronisa- } \\
\text { Synchronisation managed } \\
\text { by software }\end{array}$ \\
$\begin{array}{l}\text { Fixed window for optical } \\
\text { flow analysis }\end{array}$ & $\begin{array}{l}\text { Optical flow recording } \\
\text { based on marker detection } \\
\text { and tracking }\end{array}$ \\
$\begin{array}{l}\text { Artefacts caused by the } \\
\text { low camera resolution }\end{array}$ & HD camera \\
\hline
\end{tabular}

First experiments solving some of these issues have been recently performed in the lab with a high definition camera (Canon IXUS $220 \mathrm{HS}$ ) and based on marker tracking within the image frames. The results show promising correlation between acceleration and optical flow data (Fig. 3). The next step will be to check its intraoperative usability.

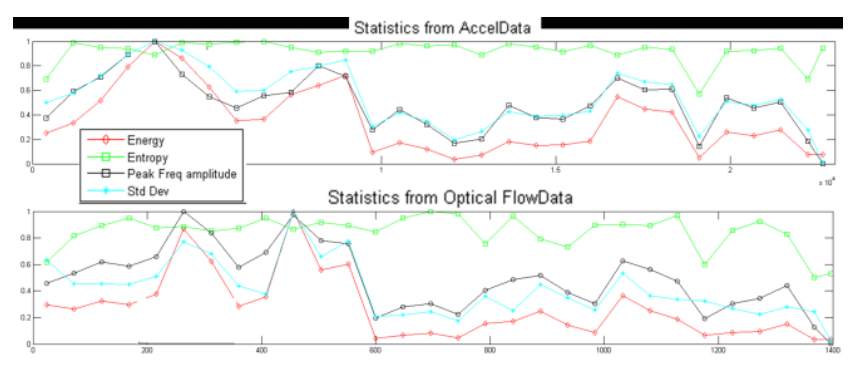

Figure 3: Comparison of optical and accelerometer raw data and extracted mathematical features over time $[\mathrm{s}]$

\section{Acknowledgement}

This research has been supported by the Swiss National Science Foundation (SNSF) and the School of Life Sciences.

\section{Bibliography}

[1] Z. Uhríková, O. Sprdlík, et al.: Validation of a new tool for automatic assessment of tremor frequency from video recordings. Journal of neuroscience methods, vol. 198(1), pp. 110-3, 2011

[2] M. Asyali, T. Dalbasti: An image analysis based method for the quantification of tremor, vol. 2(7), pp. 631-640, 2007.

[3] J. Coste,L. Ouchchane et. al.: New electrophysiological mapping combined with MRI in parkinsonian's subthalamic region, EJN, vol. 29, pp. 1627-1633, 2009

[4] B. Lucas, T. Kanade: An image registration technique with an application to stereo vision. In: Proc. of the Internat. Joint Conf. on Artificial Intelligent, pp. 674-679, 1981. 